\title{
Meryl Streep and the problems of clinical trials
}

\author{
David A Isenberg \\ See related research by Ginzler et al., http://arthritis-research.com/content/14/1/R33
}

\begin{abstract}
A clinical trial of lupus patients with nephritis was established to determine any possible role for Atacicept, a biologic drug that blocks two B-cellactivating factors (BLyS and APRIL). The trial was stopped after just six patients had been enrolled because three patients developed serious infections. Initial concerns that the biologic was the main cause of the increased susceptibility to these infections have had to be revised on close inspection of the data. The evidence clearly points to a previously unrecognised capacity for mycophenolate to cause notable drops in immunoglobulin levels as the prime suspect.
\end{abstract}

Reading the research article in the previous issue of Arthritis Research \& Therapy by Ellen Ginzler and colleagues describing the remarkable events linked to a trial of Atacicept in combination with mycophenolate and corticosteroids [1], I was reminded of the film It's Complicated starring the incomparable Meryl Streep that came out a year or so ago.

The business of performing clinical trials is complex and perhaps never more so than when a potent mix of immunosuppressive drugs is supplemented with a far more specific biologic, in the hope of getting better disease control in patients with hard-to-treat autoimmune conditions. The study that Ginzler and colleagues were seeking to carry out was to explore whether Atacicept, which blocks two B-cell-activating factors (APRIL and $\mathrm{BLyS}$ ), would be of value in patients with lupus nephritis. In this study, and prior to commencing either Atacicept or placebo therapy, the patients were treated with significant doses of mycophenolate (up to $3 \mathrm{~g} /$ day) and prednisolone (up to $60 \mathrm{mg} /$ day) for 2 weeks. Remarkably, three of the first four patients who were subsequently

*Correspondence: d.isenberg@ucl.ac.uk

Centre for Rheumatology, Division of Medicine, University College London, The Rayne Building, 5 University Street, London WC1E 6JF, UK treated with Atacicept developed profound reductions in their serum IgG levels. Patient 3 dropped their IgG level to $2.9 \mathrm{~g} / \mathrm{l}$ and developed pneumonia due to haemophilus influenzae. Patient 13 had an IgG level down to $2.5 \mathrm{~g} / \mathrm{l}$ and developed pneumonia due to Legionella pneumophila; and Patient 14 also dropped her IgG level to $2.5 \mathrm{~g} / \mathrm{l}$, subsequently developing Bacillus bacteraemia.

Although to quote Paul Simon there had been 'hints and allegations' that high-dose corticosteroids can reduce IgG synthesis [2], neither the authors of the Ginzler study nor any colleagues I have spoken to in rheumatology or nephrology could recall seeing anything like these very profound falls in IgG levels within a fortnight of starting steroids and mycophenolate. In reality, I suspect, few clinicians actually measure immunoglobulin levels in these circumstances. In addition, as Ginzler and colleagues point out, hypogammaglobulinemia has been reported in approximately one-half of renal transplant patients receiving mycophenolate and a combination of corticosteroids [3] - but again this has not, to the best of my knowledge, been reported in patients with lupus nephritis, who are these days often treated in this way. Indeed, the recent success of the Aspriva Lupus Management Study trials suggest that this combination may be used even more widely in the future $[4,5]$.

Another warning sign of impending trouble was the very low $\mathrm{CD} 4$ counts identified in those patients who went on to develop pneumonia. These CD4 levels should also be monitored.

Fortunately, there were no fatalities in this study, but two questions do emerge and are not, I think, easily answered. First, was the company simply unlucky with their choice of patients? The patients who were eventually randomised to Atacicept had high levels of proteinuria at screening, implying that they had particularly aggressive disease. These patients are likely to have received more immunosuppression previously. Patients with significant proteinuria are also likely to be leaking IgG in the urine. They therefore need higher IgG production to maintain normal serum levels, and may be sensitive to drugs that interfere with this production. In future, it is reasonable to anticipate that pharmacogenomics may provide an essential guide to which drugs will better suit which individual patients. Two of the patients whose 
immunoglobulin levels dropped so rapidly were AfricanAmericans and one was Asian. It might perhaps be relevant that differences emerged in the responses to mycophenolate and cyclophosphamide in the original Aspriva Lupus Management Study depending on ethnicity [6]. More Black and Hispanic patients therefore responded to mycophenolate than to cyclophosphamide.

Secondly, should the company have continued the study, since we are no closer to knowing whether or not Atacicept might of value in treating patients with lupus nephritis - especially those who do not respond to conventional immunosuppression? Meryl Streep has of course gone on to star in another leading role as The Iron Lady - alias Margaret Thatcher. Would the company been better served by an Iron Lady (or possibly an Iron Man!) who would have pointed out that the dramatic falls in IgG commenced prior to the introduction of Atacicept (although, admittedly, some continuing fall did take place in two of these patients after the Atacicept was started) that is, the biologic was not the initial culprit. Such an Iron Lady might have persuaded the Safety Committee that, provided the IgG level on day 1 of the trial (before Atacicept was given) was well within normal limits, there really would be no reason to stop the study, which was important and must, of course, have cost many millions of dollars to get started.

These events occurred over 3 years ago. Even allowing for the inevitable delays to review the data carefully, it seems regrettable that a novel message - that is, the importance of measuring immunoglobulin (and possibly
CD4) levels after starting patients on mycophenolate and high-dose steroids prior to the introduction of Atacicept has taken so long to be published. One wonders what Meryl Streep (in the guise of Mrs Thatcher) would have thought about this. I suspect she would not have been amused.

Competing interests

The author declares that he has no competing interests.

Published: 23 March 2012

References

1. Ginzler EM, Wax S, Rajeswaran A, Copt S, Hilson J, Romos E, Singer NG: Atacicept in combination with MMF and corticosteroids in lupus nephritis: results of a prematurely terminated study. Arthritis Res Ther 2012, 14:R33.

2. McMillan R, Longmire R, Yelenosky R: The effect of corticosteroids on human IgG synthesis. J Immunol 1976, 116:1592-1595

3. Broeden EN, Wissing HM, Hazzon M, Ghisdal L, Hoary AD, Noel C, Mascot F, Abramswicz D: Evolution of immunoglobulins and mannose binding protein levels after renal transplantation. Transplant Int 2008, 21:57-64

4. Appel GB, Contreras G, Dooley MA, Ginzler EM, Isenberg D, Jayne D, Li L-S, M Eduardo, Sánhez-Guerrero J, Solomons N, Wofsy D: Mycophenolate mofetil versus cyclophosphamide for induction treatment of lupus nephritis. JAm Soc Nephrol 2009, 20:1103-1112.

5. Dooley MA, Jayne D, Ginzler EM, Isenberg DA, Olsen NJ, Wofsy D, Eitna F, Appel G, Contreras G, Lisk L, Solomon N: Mycophenolate versus azathioprine as maintenance therapy for lupus nephritis. N Engl J Med 2011, 365:1886-1895.

6. Isenberg DA, Appel GB, Contreras G, Dooley MA, Ginzler EM, Jayne D, Sánchez-Guerrero J, Wofsy D, Yu Xueqing, Solomons N: Influence of race/ ethnicity on response to lupus nephritis treatment: the ALMS study. Rheumatology 2010, 49:128-140.

doi:10.1186/ar3751

Cite this article as: Isenberg DA: Meryl Streep and the problems of clinical trials. Arthritis Research \& Therapy 2012, 14:113. 